\title{
An Artificial Intelligence Model to Predict the Mortality of COVID-19 Patients at Hospital Admission Time Using Routine Blood Samples: Development and Validation of an Ensemble Model
}

Hoon $\mathrm{Ko}^{1^{*}}$, MSc; Heewon Chung ${ }^{1 *}$, MSc; Wu Seong Kang ${ }^{2 *}$, MD, PhD; Chul Park ${ }^{3 *}$, MD; Do Wan Kim ${ }^{4}$, MD; Seong Eun Kim ${ }^{5}$, MD; Chi Ryang Chung ${ }^{6}$, MD, PhD; Ryoung Eun $\mathrm{Ko}^{6}$, MD, PhD; Hooseok Lee ${ }^{1}$, MSc; Jae Ho Seo ${ }^{7}, \mathrm{PhD}$; Tae-Young $\mathrm{Choi}^{8}$, PhD; Rafael Jaimes ${ }^{9}, \mathrm{PhD}$; Kyung Won Kim ${ }^{10}$, MD; Jinseok Lee ${ }^{1}, \mathrm{PhD}$

\footnotetext{
${ }^{1}$ Biomedical Engineering, Wonkwang University, Iksan, Republic of Korea

${ }^{2}$ Department of Trauma Surgery, Wonkwang University Hospital, Iksan, Republic of Korea

${ }^{3}$ Department of Internal Medicine, Wonkwang University Hospital, Iksan, Republic of Korea

${ }^{4}$ Department of Thoracic and Cardiovascular Surgery, Chonnam National University Medical School, Gwangju, Republic of Korea

${ }^{5}$ Department of Internal Medicine, Chonnam National University Medical School, Gwangju, Republic of Korea

${ }^{6}$ Department of Critical Care Medicine, Samsung Medical Center, Sungkyunkwan University School of Medicine, Seoul, Republic of Korea

${ }^{7}$ Department of Biochemistry, Wonkwang University School of Medicine, Iksan, Republic of Korea

${ }^{8}$ Department of Pathology, Wonkwang University School of Medicine, Iksan, Republic of Korea

${ }^{9}$ Biotechnology and Human Systems, Lincoln Laboratory, Massachusetts Institute of Technology, Lexington, MA, United States

${ }^{10}$ Radiology and Research Institute of Radiology, Asan Medical Center, University of Ulsan College of Medicine, Seoul, Republic of Korea

*these authors contributed equally
}

\section{Corresponding Author:}

Jinseok Lee, $\mathrm{PhD}$

Biomedical Engineering

Wonkwang University

Iksan Daero

Iksan, 54538

Republic of Korea

Phone: 821638506970

Email: gonasago@gmail.com

\section{Abstract}

Background: COVID-19, which is accompanied by acute respiratory distress, multiple organ failure, and death, has spread worldwide much faster than previously thought. However, at present, it has limited treatments.

Objective: To overcome this issue, we developed an artificial intelligence (AI) model of COVID-19, named EDRnet (ensemble learning model based on deep neural network and random forest models), to predict in-hospital mortality using a routine blood sample at the time of hospital admission.

Methods: We selected 28 blood biomarkers and used the age and gender information of patients as model inputs. To improve the mortality prediction, we adopted an ensemble approach combining deep neural network and random forest models. We trained our model with a database of blood samples from 361 COVID-19 patients in Wuhan, China, and applied it to 106 COVID-19 patients in three Korean medical institutions.

Results: In the testing data sets, EDRnet provided high sensitivity (100\%), specificity (91\%), and accuracy (92\%). To extend the number of patient data points, we developed a web application (BeatCOVID19) where anyone can access the model to predict mortality and can register his or her own blood laboratory results.

Conclusions: Our new AI model, EDRnet, accurately predicts the mortality rate for COVID-19. It is publicly available and aims to help health care providers fight COVID-19 and improve patients' outcomes.

(J Med Internet Res 2020;22(12):e25442) doi: 10.2196/25442 


\section{KEYWORDS}

COVID-19; artificial intelligence; blood samples; mortality prediction

\section{Introduction}

COVID-19 is a highly contagious infection caused by SARS-CoV2. In severe cases, COVID-19 causes acute respiratory distress, multiple organ failure, and, eventually, death [1]. As of November 2020, COVID-19 cases and deaths are approaching 60 million and 1.5 million, respectively, worldwide.

In a pandemic situation, the most important issue in the management of patients diagnosed with COVID-19 is to select patients at risk of high mortality in the early period of disease and to provide appropriate treatments [2]. Particularly, the condition of patients at high risk can rapidly deteriorate. Some papers reported that deceased COVID-19 patients initially had mild symptoms but suddenly transitioned to a critical stage, leading to death [3-5]. In Italy, 75\% of deceased patients showed mild symptoms, such as fever, dyspnea, and cough, at admission to the hospital [1]. Thus, the development of a prognostic model to predict mortality as early as possible is very critical.

In this pandemic crisis, the shortage of resources and medical staff causes big problems in the health care system. Accordingly, artificial intelligence (AI) can aid in the management of COVID-19 patients. A recent research study has developed an AI prediction model of mortality based on blood test results [6]. In this study, Yan et al initially considered 73 blood-borne markers for the mortality prediction model; finally, three blood biomarkers were selected, including lactate dehydrogenase (LDH), lymphocyte, and high-sensitivity C-reactive protein (hs-CRP). This model predicted mortality with $90 \%$ accuracy based on a decision tree using an XGBoost classifier [7] to analyze feature importance.

However, Yan et al's study has drawbacks. First, the three biomarkers derived from the XGBoost-based feature selection may not be the best choices. Feature importance provides a score indicating how each feature contributes in the construction of decision trees within the model. However, due to the stochastic nature of machine learning algorithms, each feature's importance score may vary. Moreover, in decision tree algorithms, such as an XGBoost and a random forest (RF), when multiple features have the same gain during the split, a branch in a tree is made by randomly selecting features among them. Second, numerous studies have shown that the disease progression of COVID-19 is not only associated with LDH [2,8-11], lymphocyte [12,13], and hs-CRP [2,10,14-17] but also with other blood-based biomarkers, such as neutrophil counts $[16,18,19]$, albumin $[18,20,21]$, and prothrombin activity
[18,22-24]. In our study, we developed an AI model using 28 biomarkers for predicting the mortality of COVID-19 patients. Third, the three biomarker-based AI models [6] predicted mortality 10 days before a patient's recovery or death. These limitations show that the model may not work for COVID-19 patients who have just been diagnosed and hospitalized.

Therefore, in this study, we aimed to develop an AI model based on a blood test for mortality prediction at the early stage of hospital admission. We deployed the developed AI model on a public website so that all patients and medical staff could predict mortality using individual patient blood test results.

\section{Methods}

\section{Data Sets}

This study was approved by Wonkwang University Hospital (WKUH), Chonnam National University Hospital (CNUH), and Samsung Medical Center (SMC) in Korea. Informed consent was waived. For training data, we used the blood test results obtained from 375 COVID-19 patients collected between January 10, 2020, and February 24, 2020, in Tongji Hospital, Wuhan, China [6]. Of these, 14 patients without a blood test within 1 day after the hospital admission were excluded, and 361 patients-212 males $(58.7 \%)$ and 149 females $(41.3 \%)$; mean age 58.9 years (SD 16.5)—were included. As presented in Multimedia Appendix 1, the training data set of 361 patients included the admission date and time, discharge date and time, age, gender, mortality outcome, and results of blood tests obtained within 24 hours after hospital admission. For testing data, we collected medical records on COVID-19 patients $(\mathrm{N}=106)$ from three medical institutions: CNUH $(85 / 106$, $80.2 \%)$, WKUH $(11 / 106,10.4 \%)$, and SMC (10/106, 9.4\%). The blood laboratory results from these 106 COVID-19 patients were collected between February 2020 and July 2020. Similar to the training data, we used the blood test data obtained within 24 hours after hospital admission (see Multimedia Appendix 2). For summarizing the statistics of the training and testing data sets, the patients were classified into a survivor group and a deceased group in the training and testing data sets. The number of blood tests differed across patients and institutions. The mean numbers of blood tests per patient were 61.21 (range 24-73) in the training data set and 35.36 (range 30-55) in the testing data set. The mean numbers of hospitalization days were 13.82 (survivor group) and 8.16 (deceased group) in the training data set and 18.21 (survivor group) and 17.98 (deceased group) in the testing data set (see Table 1). 
Table 1. Statistical summary of the training and testing data sets.

\begin{tabular}{|c|c|c|c|c|c|}
\hline \multirow[t]{2}{*}{ Patient data } & \multirow{2}{*}{$\begin{array}{l}\text { Training data set }(\mathrm{N}=361) \\
\text { Tongji Hospital }\end{array}$} & \multicolumn{4}{|c|}{ Testing data set $(\mathrm{N}=106)$} \\
\hline & & $\mathrm{CNUH}^{\mathrm{a}}(\mathrm{n}=85)$ & $\mathrm{WKUH}^{\mathrm{b}}(\mathrm{n}=11)$ & $\operatorname{SMC}^{\mathrm{C}}(\mathrm{n}=10)$ & Total $(\mathrm{N}=106)$ \\
\hline \multicolumn{6}{|l|}{ Number of patients, n (\%) } \\
\hline Total & $361(100)$ & $85(100)$ & $11(100)$ & $10(100)$ & $106(100)$ \\
\hline Survived & $195(54.0)$ & $85(100)$ & $9(82)$ & $10(100)$ & $104(98.1)$ \\
\hline Deceased & $212(58.7)$ & $0(0)$ & $2(18)$ & $0(0)$ & $2(1.9)$ \\
\hline \multicolumn{6}{|l|}{ Gender, n (\%) } \\
\hline Male & $212(58.7)$ & $34(40)$ & $5(45)$ & $3(30)$ & $42(39.6)$ \\
\hline Female & $149(41.3)$ & $51(60)$ & $6(55)$ & $7(70)$ & $64(60.4)$ \\
\hline \multicolumn{6}{|c|}{$\begin{array}{l}\text { Number of hospitalization } \\
\text { days, mean (SD) }\end{array}$} \\
\hline Survived & $13.82(6.38)$ & $15.06(7.90)$ & $28.16(11.13)$ & $30.95(23.03)$ & $18.21(11.46)$ \\
\hline Deceased & $8.16(7.38)$ & $\mathrm{N} / \mathrm{A}^{\mathrm{d}}$ & $17.98(11.83)$ & N/A & $17.98(11.83)$ \\
\hline Age in years, mean (SD) & $58.91(16.49)$ & $44.14(21.81)$ & $56.27(23.00)$ & $58.20(21.05)$ & $46.73(22.28)$ \\
\hline \multicolumn{6}{|c|}{$\begin{array}{l}\text { Number of blood biomarkers } \\
\text { collected }\end{array}$} \\
\hline Min-max & $24-73$ & $32-55$ & $30-52$ & $30-40$ & $30-55$ \\
\hline Mean (SD) & $61.21(6.92)$ & $36.89(4.11)$ & $35.00(5.31)$ & $34.20(3.16)$ & $35.36(4.19)$ \\
\hline
\end{tabular}

${ }^{\mathrm{a} C N U H: ~ C h o n n a m ~ N a t i o n a l ~ U n i v e r s i t y ~ H o s p i t a l . ~}$

${ }^{\mathrm{b}}$ WKUH: Wonkwang University Hospital.

${ }^{\mathrm{c}} \mathrm{SMC}$ : Samsung Medical Center.

${ }^{\mathrm{d}} \mathrm{N} / \mathrm{A}$ : not applicable; there were no deceased patients in the testing data set at this institution.

\section{Feature Selection}

Given the total 73 blood biomarkers from the training data, we performed an analysis of variance (ANOVA), which uses an $F$ test to check for any significant difference between the two groups (ie, deceased vs survivor) according to each blood biomarker. For the feature selection, we also considered the available data rate (ADR), which refers to how much blood biomarker data were available for training the AI model. This is calculated as

$$
A D R(\%)=\frac{N_{\text {biomarker }}}{N_{\text {patients }}} \times 100
$$

where $N_{\text {patients }}$ is the total number of patients $(\mathrm{N}=361)$ and $N_{\text {biomarker }}$ is the number of patients having each of the specific biomarker data.

Based on the ANOVA, we first selected the top 32 biomarkers corresponding to $P$ values less than $10^{-5}$. Subsequently, we excluded four biomarkers with ADR values of less than $90 \%$. Table 2 summarizes the final selection of 28 biomarkers with the corresponding ANOVA $P$ values and ADR values. The ANOVA $P$ values and ADR values for all 73 biomarkers in the training data set are summarized in Multimedia Appendix 3, Table S1. The sample distributions of the selected 28 biomarkers in the survivor and deceased groups are presented in Multimedia Appendix 3, Figure S1. 
Table 2. List of 28 blood biomarkers selected for the artificial intelligence model training.

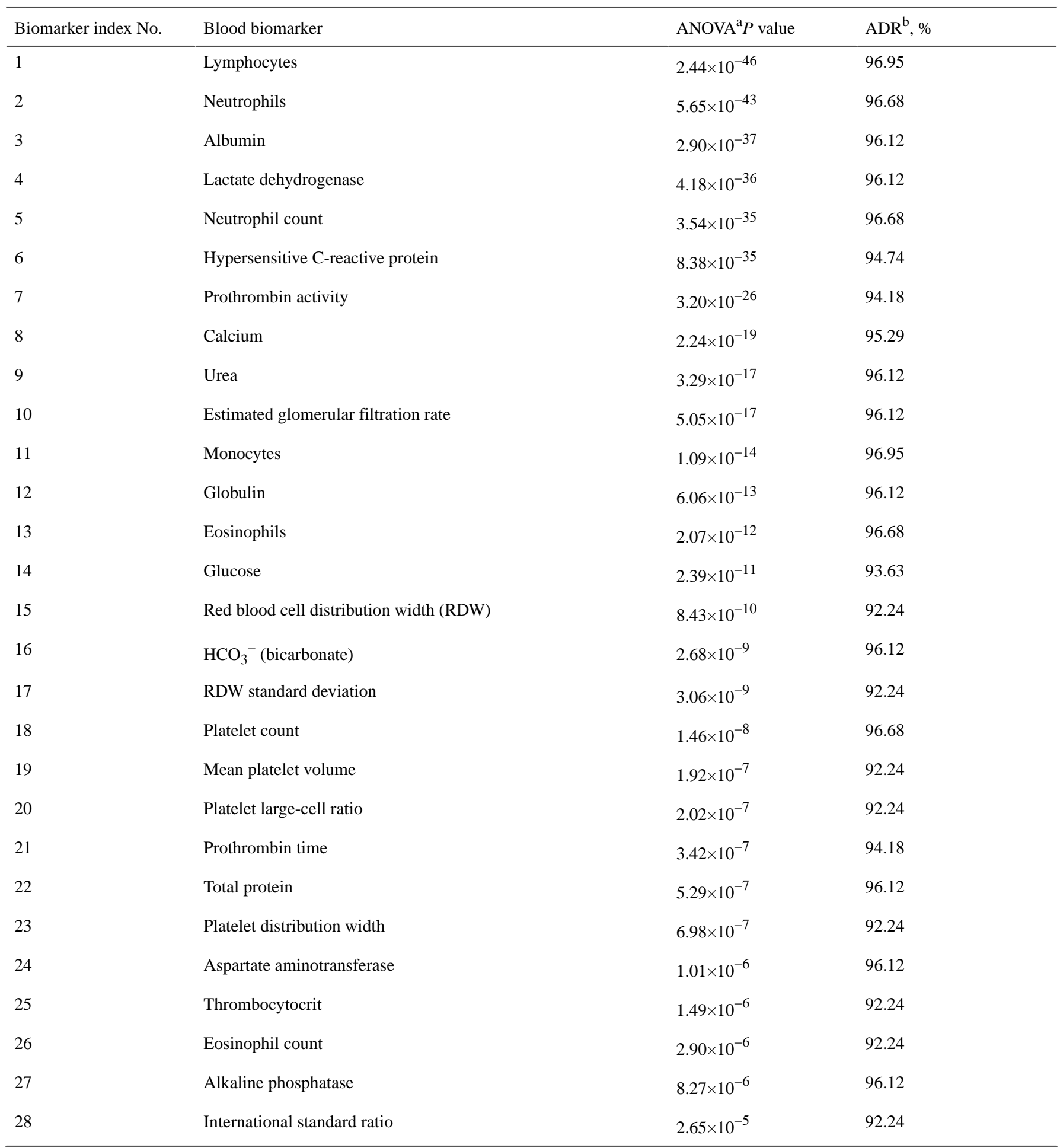

aNOVA: analysis of variance.

${ }^{\mathrm{b}} \mathrm{ADR}$ : available data rate.

\section{Preprocessing}

Given the selected 28 biomarkers, the mean number of available biomarkers per patient was 27.22 (SD 2.33) for the training data and 16.86 (SD 1.58) for the testing data, as summarized in Table
3. To handle the missing data, we calculated the mean value from the training data for each biomarker and replaced the missing data with the mean value for the training and testing data sets. We then added two more features (ie, age and gender) to the 28 biomarkers and trained our AI model using 30 features. 
Table 3. Number of available blood biomarkers per patient for the artificial intelligence model training.

\begin{tabular}{ll}
\hline Data sets and sources & $\begin{array}{l}\text { Number of blood biomarkers } \\
\text { Mean (SD) }\end{array}$ \\
\hline Training data: Tongji Hospital & $27.22(2.33)$ \\
Testing data & $20.39(1.13)$ \\
$\quad$ Chonnam National University Hospital & $13-28$ \\
$\quad$ Wonkwang University Hospital & $19.82(1.94)$ \\
$\quad$ Samsung Medical Center & $14.40(1.58)$ \\
Total & $16.86(1.58)$ \\
\hline
\end{tabular}

With the 30 features, we performed data set standardization, which is a common requirement for machine learning estimators. The standardization changes the data distribution of each feature with zero mean and standard deviation of 1 as

Data $_{\text {standard }}=\frac{\text { Data }- \text { mean }(\text { train })}{S D(\text { train })}$,

where mean(train) and $S D$ (train) are the mean and standard deviation values, respectively, for each feature from the training data. The standardization was applied to the training and testing data sets.

\section{Development of an Ensemble AI Model}

As illustrated in Figure 1, the new ensemble AI model is composed of a 5-layer deep neural network (DNN) and RF model. Our ensemble AI model was named as EDRnet (ensemble learning model based on DNN and RF models). The 5-layer DNN was comprised of an input layer, three fully connected (FC) layers, and an output layer. The input layer contained 30 features, including 28 biomarkers, age, and gender. The input layer was fed into three FC layers in a series, each of which consisted of 30,16 , and 8 nodes. To alleviate the overfitting issue, we applied a dropout rate of 0.3 . Then, the last FC layer was fed into a softmax layer, which is an output layer providing the probabilities for the patient mortality. Figure S2 in Multimedia Appendix 3 shows our DNN model and its printed textual summary run on Keras, where the total number of parameters (ie, weights and biases) was 1571 . 
Figure 1. Proposed ensemble model (EDRnet) composed of a 5-layer deep neural network (DNN) and random forest (RF) model for the mortality prediction. In the training of both models, a 10-time-repetition 10-fold stratified cross-validation was separately performed, and the predicted mortality probabilities of the DNN model, $\mathrm{p}(\mathrm{DNN})$, and the RF model, $\mathrm{p}(\mathrm{RF})$, were calculated. The final predicted mortality probability of the ensemble model, $\mathrm{p}(\mathrm{EDR})$, was obtained by soft voting based on the $\mathrm{p}(\mathrm{DNN})$ and the $\mathrm{p}(\mathrm{RF})$. ADR: available data rate; ANOVA: analysis of variance; EDRnet: ensemble learning model based on DNN and RF models.

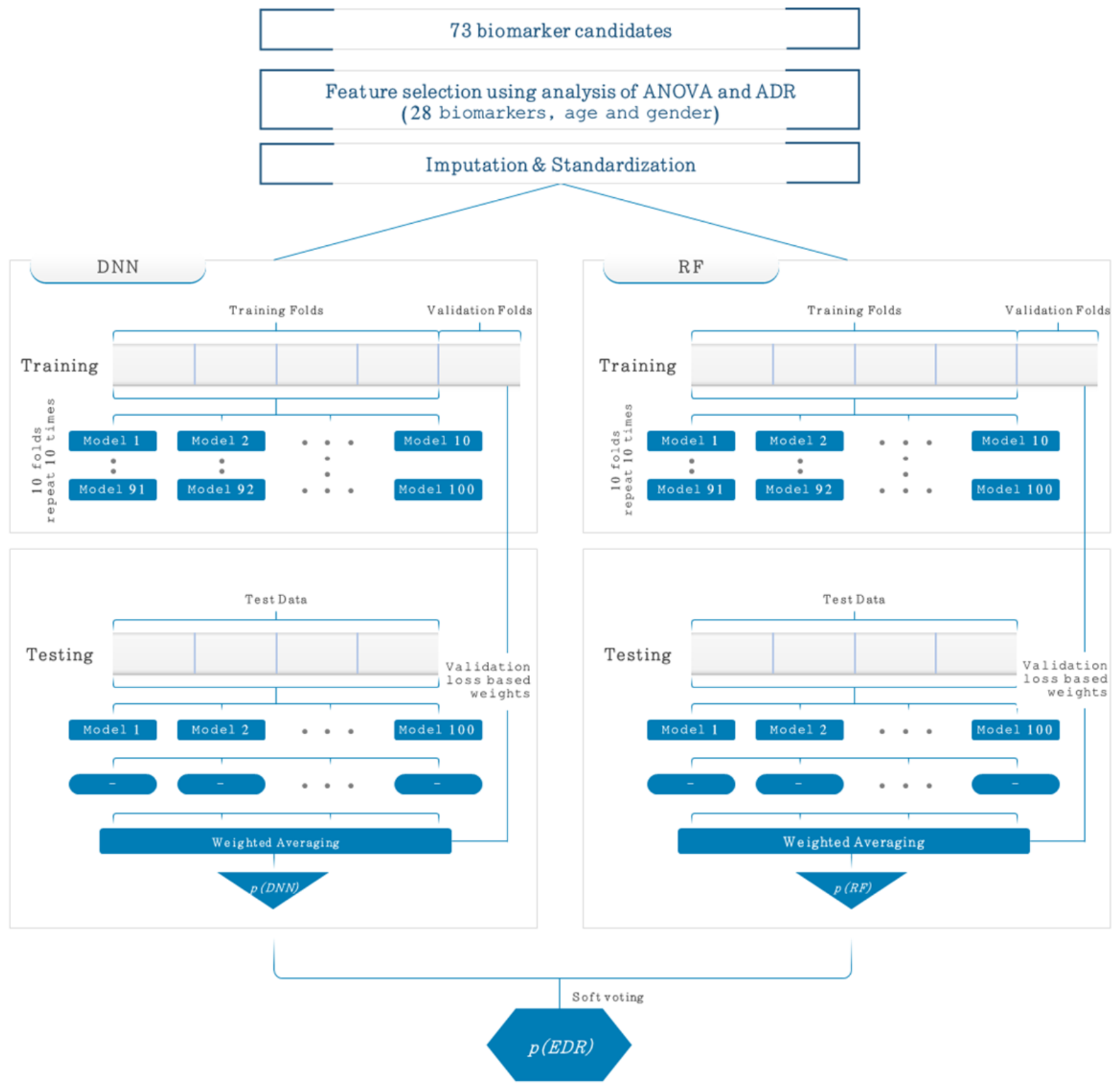

For the 5-layer DNN, a 10-time-repetition 10-fold stratified cross-validation was performed to confirm the model's generalization ability. The training data $(\mathrm{N}=361)$ were randomly shuffled and partitioned into 10 equal subgroups in a stratified manner. Of the 10 subgroups, a single subgroup was retained as the validation data set for testing the model, and the remaining nine subgroups were used as the training data set. The process was then repeated 10 times, with each of the 10 subgroups used exactly once as the validation data set. By repeating this stratified 10 -fold cross-validation process 10 times, a total of 100 models from the 5-layer DNN were derived. Then, we ensembled the models with the weighted average as

$p(D N N)=\bar{w}_{m}(D N N) \cdot p_{m}(D N N), \quad m=1,2, \ldots, 100$, where $p_{m}(D N N)$ is the predicted mortality probability value from the $m^{\text {th }}$ model of the DNN, $p(D N N)$ is the ensemble result corresponding to the predicted mortality prediction probability, and $\widetilde{w}_{m}(D N N)$ is the normalized weight value for the $m^{\text {th }}$ model. We obtained the normalized weight value $\widetilde{w}_{m}(D N N)$ as

$$
(D N N)=\frac{w_{m}(D N N)}{\sum_{m=1}^{100} w_{m}(D N N)},
$$

where the weight $w_{m}(D N N)$ was obtained using the validation loss from the $m^{\text {th }}$ model, $l_{m}(D N N)$, as 
$w_{m(D N N)}=\frac{\min \left(l_{m}(D N N)\right)}{l_{m}(D N N)}$.

Along with the 5-layer DNN, we separately trained an RF model. For the RF model, 100 decision trees were trained with a maximum depth of 4 and maximum feature number of 5 . Similar to the 5-layer DNN, we performed a 10-time-repetition 10-fold stratified cross-validation and ensembled the 100 models with the weighted average as

$p(R F)=\bar{w}_{m}(R F) \cdot p_{m}(R F), \quad m=1,2, \ldots, 100$,

where $p_{m}(R F)$ is the predicted mortality probability value from the $m^{\text {th }}$ model of the RF, $p(R F)$ is the ensemble result corresponding to the predicted mortality prediction probability, and $\vec{w}_{m}(R F)$ is the normalized weight value for the $m^{\text {th }}$ model.

We obtained the normalized weight value $\widetilde{w}_{m}$ as

$\bar{w}_{m}(R F)=\frac{w_{m}(R F)}{\sum_{m=1}^{100} w_{m}(R F)}$

where the weight $w_{m}(R F)$ was obtained using the validation loss from the $m^{\text {th }}$ model, $l_{m}(R F)$, as

$w_{m}(R F)=\frac{\min \left(l_{m}(R F)\right)}{l_{m}(R F)}$.

Given the two ensemble results $p(D N N)$ and $p(R F)$, we finally obtained the final predicted mortality probability value using soft voting. Based on the average of the two probability values $p(D N N)$ and $p(R F)$, if the value is greater than or equal to 0.5 , then the prediction result represents death; otherwise, it represents survival.

\section{Implementation}

We implemented and trained EDRnet using TensorFlow, version 1.13.1 for graphics processing unit (GPU), and Keras, version 2.2.4 for GPU. NumPy, version 1.16.4; Pandas, version: 0.25.3; Matplotlib, version 3.1.2; and scikit-learn, version 0.22.1, were used to build the model and analyze the results. We trained the models with the Adam optimizer and a binary cross-entropy cost function in equation 9 with a learning rate of 0.0001 and a batch size of 64 on the NVIDIA GeForce GTX 1080 Ti GPU as

$$
B C E(x)=-\frac{1}{N} \sum_{i=1}^{N} y_{i} \log \left(p\left(y_{i}\right)\right)+\left(1-y_{i}\right) \log \left(1-p\left(y_{i}\right)\right),
$$

where $y_{i}$ is the label (ie, 1 for deceased and 0 for survived) and $p\left(y_{i}\right)$ is the predicted probability of each patient being deceased for the batch size $N$ number of patients.

\section{Performance Evaluation of AI Models}

To evaluate the performance of the AI models in predicting mortality, we used the sensitivity, specificity, accuracy, and balanced accuracy metrics, defined as

$$
\begin{aligned}
& \text { Sensitivity }=\frac{T P}{T P+F N}, \quad(10) \\
& \text { Specificity }=\frac{T N}{T N+F P}, \quad(11) \\
& \text { Accuracy }=\frac{T P+T N}{T P+T N+F P+F N},
\end{aligned}
$$

Balanced Accuracy $=\frac{\text { Sensitivity }+ \text { Specificity }}{2}$,

where TP, TN, FP, and FN represent the true positive, true negative, false positive, and false negative, respectively.

In the training data set, the prediction performance of the AI models was evaluated based on a 10-time-repetition 10-fold stratified cross-validation. In the testing data set, the prediction performance of the AI models was independently evaluated.

To compare the performance of our proposed EDRnet model with those of other external AI models, we separately trained the models of XGBoost and AdaBoost (AB), each of which was evaluated as a single model and as an ensemble model combined with DNN, resulting in four models: XGBoost, $\mathrm{AB}$, ensemble with DNN and XGBoost (EDX), and ensemble with DNN and AB (EDA). For the training of these models, we searched the optimal hyperparameters providing the highest validation accuracy value, as presented in Multimedia Appendix 3, Table $\mathrm{S} 2$. In addition, we adopted a recently published AI model by Li et al [6] using a decision tree via an XGBoost-based feature selection for performance comparison. All five external AI models were evaluated using our testing data set of 106 patients.

\section{Results}

The cross-validation of RF, DNN, and our ensemble model EDRnet showed that the accuracy on the validation data set is $89 \%$ for RF, $92 \%$ for DNN, and 93\% for EDRnet. Thus, EDRnet provides the highest sensitivity, specificity, accuracy, and balanced accuracy values (see Table 4).

Table 4. Cross-validation accuracy comparison.

\begin{tabular}{lllll}
\hline Model & \multicolumn{2}{l}{ Cross-validation results $(\mathrm{N}=361)$, mean $(\mathrm{SD})$} & & Bccuracy \\
& Sensitivity & Specificity & $0.89(0.04)$ & $0.89(0.04)$ \\
\hline Random forest & $0.89(0.06)$ & $0.89(0.07)$ & $0.92(0.04)$ & $0.92(0.06)$ \\
Deep neural network & $0.91(0.06)$ & $0.93(0.04)$ & $0.93(0.03)$ & $0.93(0.05)$ \\
EDRnet $^{\mathrm{a}}$ & $0.92(0.05)$ & $0.93(0.03)$ & & \\
\hline
\end{tabular}

${ }^{a}$ EDRnet: ensemble learning model based on deep neural network and random forest models. 
Moreover, we applied EDRnet to 106 Korean patients as an independent testing data set to check the TP, TN, FP, FN, sensitivity, specificity, accuracy, and balanced accuracy. The results show a sensitivity of $100 \%$, specificity of $91 \%$, accuracy of $92 \%$, and balanced accuracy of $96 \%$, indicating that the model trained and validated on Chinese patient data can be applied to Korean patients for mortality prediction (see Table 5). The computational times of DNN and RF in EDRnet for the training were 796 and 126 seconds, respectively. The overall computational time for the testing of EDRnet was 72 seconds.

Table 5. Test results from our proposed EDRnet (ensemble learning model based on deep neural network and random forest models) model.

\begin{tabular}{|c|c|c|c|c|c|c|c|c|c|}
\hline Model & Testing data & $\begin{array}{l}\text { True negative, } \\
\%\end{array}$ & $\begin{array}{l}\text { False positive, } \\
\%\end{array}$ & $\begin{array}{l}\text { False negative, } \\
\%\end{array}$ & $\begin{array}{l}\text { True positive, } \\
\%\end{array}$ & Sensitivity & Specificity & Accuracy & $\begin{array}{l}\text { Balanced } \\
\text { accuracy }\end{array}$ \\
\hline EDRnet & $\mathrm{CNUH}^{\mathrm{a}}$ & 79 & 6 & 0 & 0 & $\mathrm{~N} / \mathrm{A}^{\mathrm{b}}$ & 0.93 & 0.93 & 0.93 \\
\hline EDRnet & WKUH $^{\mathrm{c}}$ & 7 & 2 & 0 & 2 & 1.00 & 0.78 & 0.82 & 0.89 \\
\hline EDRnet & $\mathrm{SMC}^{\mathrm{d}}$ & 9 & 1 & 0 & 0 & N/A & 0.90 & 0.90 & 0.90 \\
\hline EDRnet & Total & 95 & 9 & 0 & 2 & 1.00 & 0.91 & 0.92 & 0.96 \\
\hline
\end{tabular}

${ }^{\mathrm{a} C N U H:}$ Chonnam National University Hospital.

${ }^{\mathrm{b}} \mathrm{N} / \mathrm{A}$ : not applicable.

${ }^{\mathrm{c}}$ WKUH: Wonkwang University Hospital.

${ }^{\mathrm{d}}$ SMC: Samsung Medical Center.

Next, we summarized the performance comparison results between XGBoost, AB, RF, DNN, EDX, EDA, Li et al's model [6], and EDRnet. Considering all variables, EDRnet provided the highest prediction performance. Indeed, the balanced accuracy was $88 \%$ with XGBoost, $89 \%$ with $\mathrm{AB}, 92 \%$ with RF
$71 \%$ with DNN, $88 \%$ with EDX, $71 \%$ with EDA, $67 \%$ with Li et al's model [6], and $96 \%$ with EDRnet. Notably, the accuracy of Li et al's model was only $36 \%$, indicating that a few blood markers may not be sufficient to predict patient mortality (see Table 6).

Table 6. Comparison of the performance of various methods.

\begin{tabular}{|c|c|c|c|c|c|c|c|c|}
\hline Model & $\begin{array}{l}\text { True negative, } \\
\%\end{array}$ & $\begin{array}{l}\text { False positive, } \\
\%\end{array}$ & $\begin{array}{l}\text { False negative, } \\
\%\end{array}$ & $\begin{array}{l}\text { True positive, } \\
\%\end{array}$ & Sensitivity & Specificity & Accuracy & $\begin{array}{l}\text { Balanced } \\
\text { accuracy }\end{array}$ \\
\hline XGBoost & 80 & 24 & 0 & 2 & 1.00 & 0.77 & 0.77 & 0.88 \\
\hline AdaBoost & 81 & 23 & 0 & 2 & 1.00 & 0.78 & 0.78 & 0.89 \\
\hline Random forest & 87 & 17 & 0 & 2 & 1.00 & 0.84 & 0.84 & 0.92 \\
\hline $\begin{array}{l}\text { 5-layer deep neural net- } \\
\text { work (DNN) }\end{array}$ & 95 & 9 & 1 & 1 & 0.50 & 0.91 & 0.90 & 0.71 \\
\hline DNN + XGBoost & 80 & 24 & 0 & 2 & 1.00 & 0.77 & 0.77 & 0.88 \\
\hline DNN + AdaBoost & 96 & 8 & 1 & 1 & 0.50 & 0.92 & 0.91 & 0.71 \\
\hline Li et al's model [6] & 36 & 68 & 0 & 2 & 1.00 & 0.35 & 0.36 & 0.67 \\
\hline $\begin{array}{l}\text { DNN + random forest } \\
\left(\text { EDRnet }^{\mathrm{a}}\right)\end{array}$ & 95 & 9 & 0 & 2 & 1.00 & 0.91 & 0.92 & 0.96 \\
\hline
\end{tabular}

${ }^{a}$ EDRnet: ensemble learning model based on DNN and random forest models.

Our proposed EDRnet model used 28 blood biomarkers for prediction, but it does not require all 28 blood biomarkers. In our testing data sets, EDRnet training was validated using available biomarkers, ranging from 14 to 24 , for each patient (see Figure 2). The results reveal that the majority of the patients had 19 to 21 available biomarkers (ie, 19 in 15 patients, 20 in
41 patients, and 21 in 22 patients) with a similarly high prediction accuracy (ie, $93 \%, 95 \%$, and $86 \%$, respectively). For the patients with 17 and 18 available biomarkers, the accuracy was $75 \%$ and $50 \%$, respectively. By contrast, the patients with 14 to 16 biomarkers showed a high accuracy ranging from $83 \%$ to $100 \%$. 
Figure 2. Accuracy with the number of available blood biomarkers from the 106-patient testing data set.

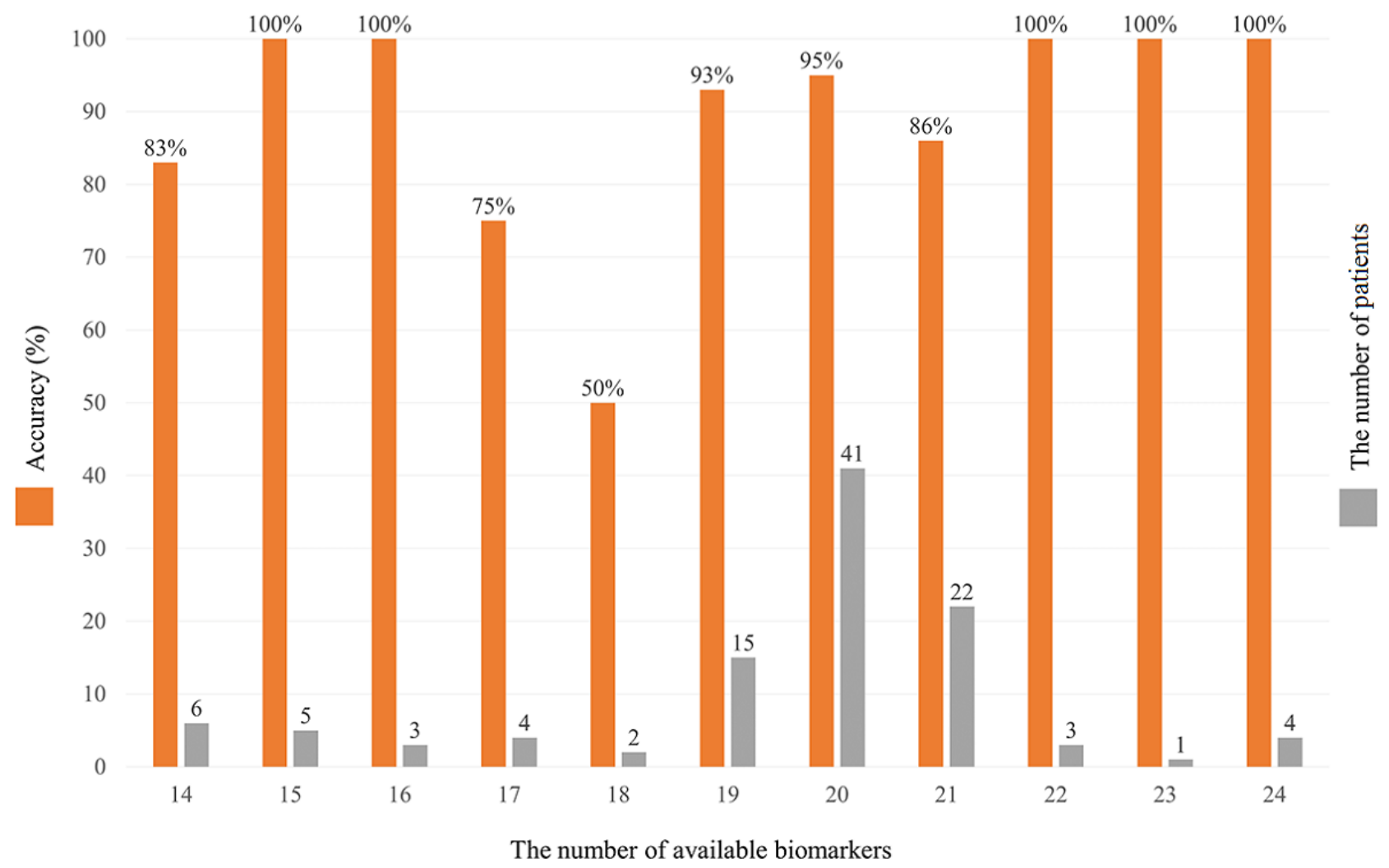

To further investigate the effect of the number of available biomarkers, we estimated the accuracy values according to the number of available biomarkers (see Figure 3). For the estimation, we randomly selected 1 to 20 biomarkers from all of the testing data points and tested the model with a 100-time where the actual available number of biomarkers was equal to or greater than the number of randomly selected biomarkers were simulated. The results show that accuracy increases with the number of available biomarkers until reaching 19 repetition. When randomly selecting biomarkers, only samples biomarkers.

Figure 3. Estimated accuracy values according to the number of available biomarkers. Red circles represent the median. The bars at the top and bottom represent the 75th and 25th percentiles, respectively. The blue rectangles at the top and bottom represent the 90th and 10th percentiles, respectively. The blue diamonds at the top and bottom represent the 95th and 5th percentiles, respectively.

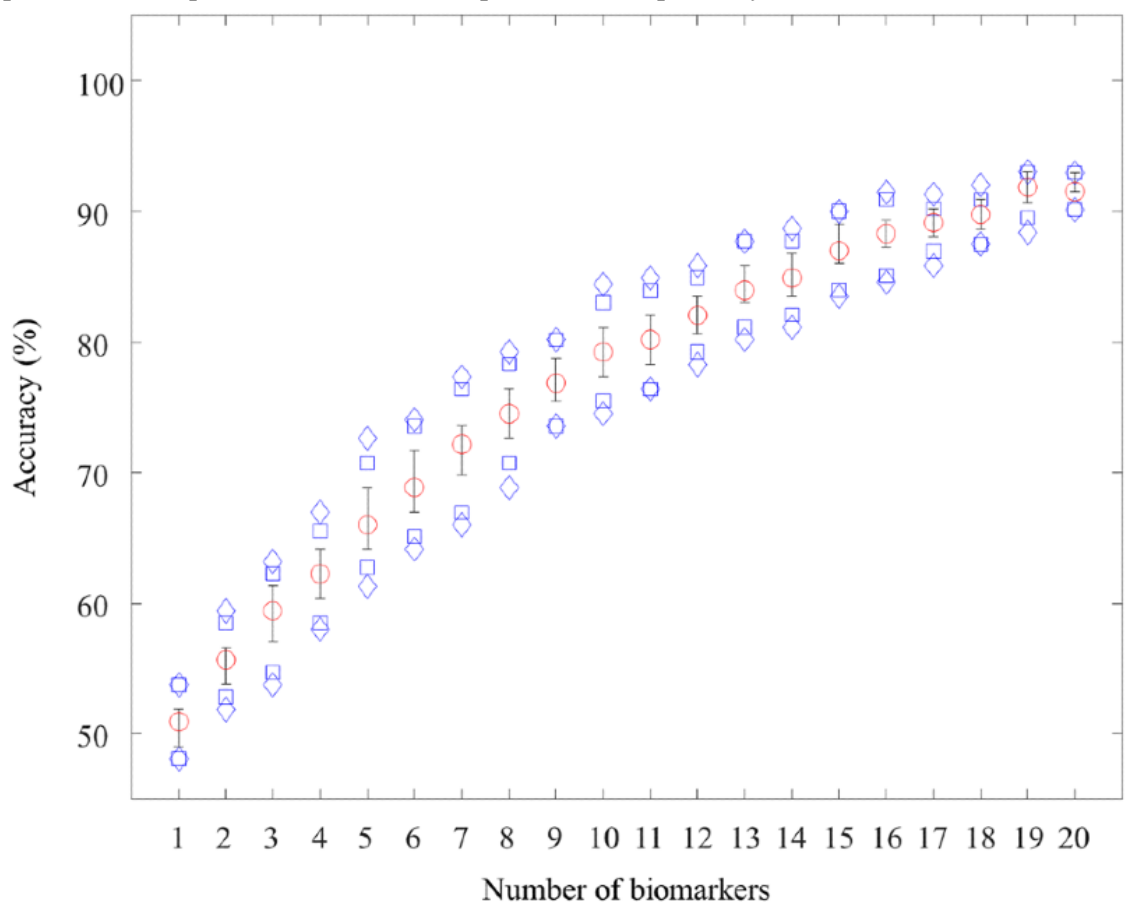


Furthermore, our developed AI model, EDRnet, was successfully deployed on a public website [25] so that anyone can predict mortality using individual blood test results. The web application provides predicted mortality probability, as shown in Figure 4. A user inputs his or her blood sample results (see Figure 4a), and then the predicted mortality results are presented (see Figure 4b). Currently, the web application does not store any information entered by users. However, we consider and plan to store information entered by users on agreement to improve the AI model via a real-time learning process.

Figure 4. Deployed web application, BeatCOVID19 [25]: (a) input windows where a user inputs his or her blood sample results and (b) the predicted mortality results after entering the blood sample results.

(a)

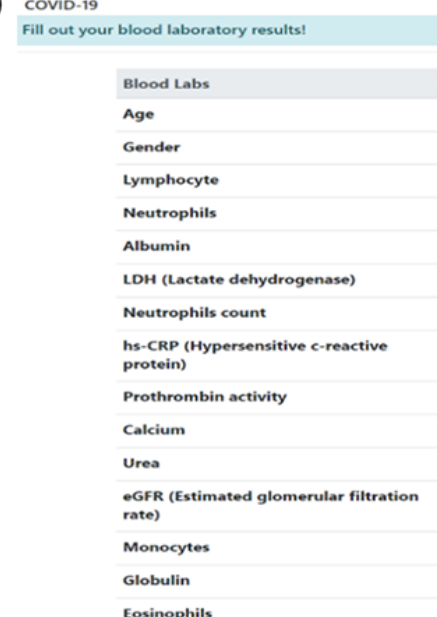

(b)

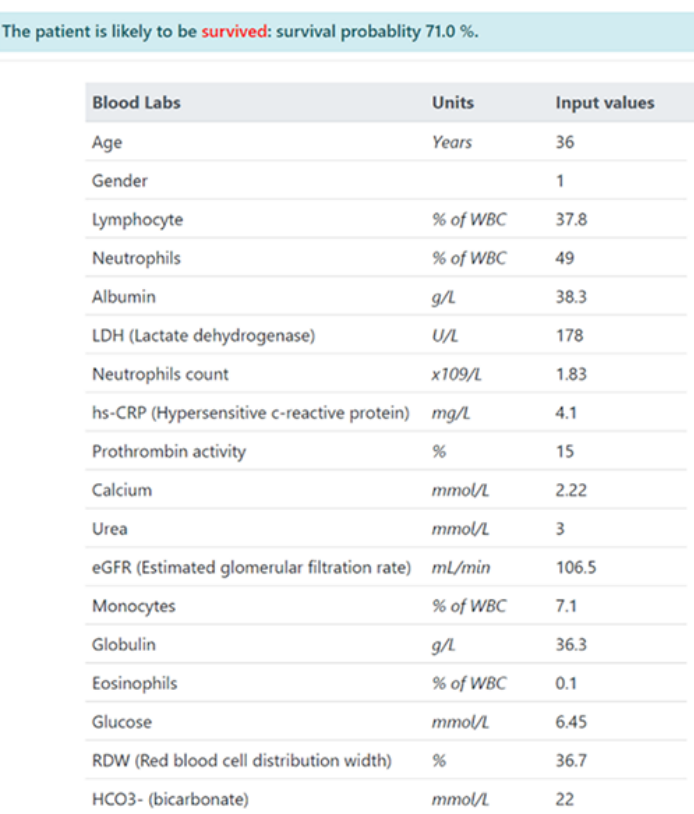

Regarding clinical characteristics (see Table 7), there were no significant differences in comorbidity. In terms of initial symptoms, the deceased group had more frequent dyspnea symptoms than the survivor group $(66.7 \%$ vs $16.8 \% ; P=.04)$. All patients from the deceased group required oxygen supply. The deceased group had more frequent altered mentality than the survivor group $(50.0 \%$ vs $1.0 \% ; P=.02)$. There was no

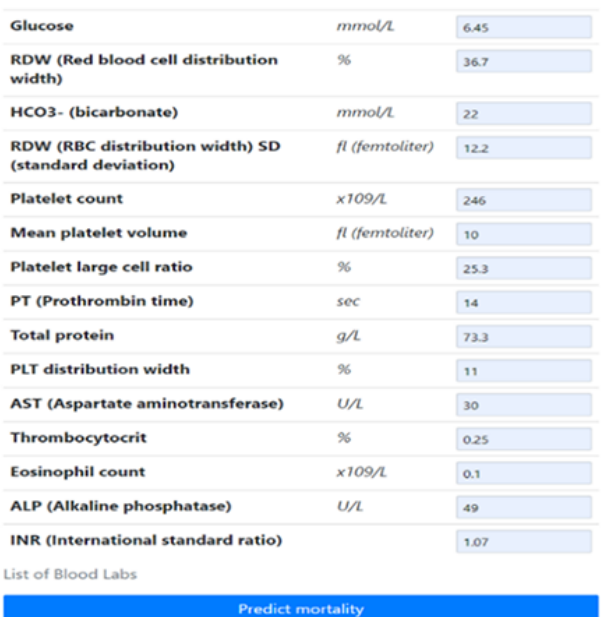

\begin{tabular}{lll}
\hline RDW (Red blood cell distribution width) & $\%$ & 36.7 \\
\hline HCO3- (bicarbonate) & mmol/L & 22 \\
\hline $\begin{array}{l}\text { RDW (RBC distribution width) SD (standard } \\
\text { deviation) }\end{array}$ & fl (femtoliter) & 12.2 \\
\hline Platelet count & $x 109 / \mathrm{L}$ & 246 \\
\hline Mean platelet volume & $\mathrm{fl}($ (femtoliter) & 10 \\
\hline Platelet large cell ratio & $\%$ & 25.3 \\
\hline PT (Prothrombin time) & $\mathrm{sec}$ & 14 \\
\hline Total protein & $\mathrm{g} / \mathrm{L}$ & 73.3 \\
\hline PLT distribution width & $\%$ & 11 \\
\hline AST (Aspartate aminotransferase) & $\mathrm{U} / \mathrm{L}$ & 30 \\
\hline Thrombocytocrit & $\%$ & 0.25 \\
\hline Eosinophil count & $x 109 / \mathrm{L}$ & 0.1 \\
\hline ALP (Alkaline phosphatase) & $\mathrm{U} / \mathrm{L}$ & 49 \\
\hline INR (International standard ratio) & & 1.07 \\
\hline
\end{tabular}

significant difference in terms of antiviral drugs (ie, lopinavir or ritonavir, chloroquine or hydroxychloroquine, ribavirin, remdesivir, and oseltamivir) or anti-inflammatory drugs (ie, interferon, dexamethasone, and methylprednisolone) between the deceased and survivor groups. However, the deceased group received more antibiotics or combination therapy. 
Table 7. Clinical characteristics of the patient groups from the testing data set.

\begin{tabular}{|c|c|c|c|c|}
\hline Characteristics & Deceased group $(\mathrm{n}=2)$ & Survivor group $(n=104)$ & Total $(\mathrm{N}=106)$ & $P$ value \\
\hline \multicolumn{5}{|l|}{ Comorbidity, n (\%) } \\
\hline Diabetes mellitus & $0(0)$ & $10(9.6)$ & $10(9.4)$ & $>.99$ \\
\hline Asthma & $0(0)$ & $8(7.7)$ & $8(7.5)$ & $>.99$ \\
\hline Chronic obstructive pulmonary disease & $0(0)$ & $0(0)$ & $0(0)$ & $>.99$ \\
\hline Coronary heart disease & $0(0)$ & $3(2.9)$ & $3(2.8)$ & $>.99$ \\
\hline Cardiovascular disease & $0(0)$ & $1(1.0)$ & $1(0.9)$ & $>.99$ \\
\hline Chronic kidney disease & $0(0)$ & $1(1.0)$ & $1(0.9)$ & $>.99$ \\
\hline Chronic liver disease & $0(0)$ & $0(0)$ & $0(0)$ & $>.99$ \\
\hline Congestive heart failure & $1(50)$ & $3(2.9)$ & $4(3.8)$ & .11 \\
\hline Cancer & $0(0)$ & $3(2.9)$ & $3(2.8)$ & $>.99$ \\
\hline \multicolumn{5}{|l|}{ Initial symptom, n (\%) } \\
\hline Fever & $2(100)$ & $59(56.7)$ & $61(57.5)$ & .61 \\
\hline Cough & $1(50)$ & $46(44.2)$ & $47(44.3)$ & $>.99$ \\
\hline Dyspnea & $2(100)$ & $18(16.8)$ & $20(18.2)$ & .04 \\
\hline Diarrhea & $0(0)$ & $11(10.3)$ & $11(10.0)$ & $>.99$ \\
\hline Myalgia & $0(0)$ & $26(24.3)$ & $26(23.6)$ & $>.99$ \\
\hline \multicolumn{5}{|l|}{ Initial vital sign, mean (SD) } \\
\hline Systolic blood pressure (mm Hg) & $127.5(17.7)$ & $128.0(18.5)$ & $128.0(18.4)$ & .97 \\
\hline Diastolic blood pressure (mm Hg) & $74.5(10.6)$ & $78.8(12.8)$ & $78.7(12.8)$ & .64 \\
\hline Heart rate (per minute) & $96.5(24.7)$ & $84.2(17.7)$ & $84.4(17.8)$ & .34 \\
\hline Respiration rate (per minute) & $29.5(10.6)$ & $20.2(4.1)$ & $20.4(4.3)$ & .43 \\
\hline Altered mentality, n (\%) & $1(50)$ & $1(1.0)$ & $2(1.9)$ & .02 \\
\hline \multicolumn{5}{|l|}{ Oxygen requirement, n (\%) } \\
\hline No oxygen supply & $0(0)$ & $83(79.8)$ & $83(78.3)$ & .07 \\
\hline Conventional oxygen & $1(50)$ & $15(14.4)$ & $16(15.1)$ & .69 \\
\hline High-flow nasal cannula & $0(0)$ & $3(2.9)$ & $3(2.8)$ & $>.99$ \\
\hline Noninvasive ventilation & $0(0)$ & $0(0)$ & $0(0)$ & $>.99$ \\
\hline Mechanical ventilation & $1(50)$ & $3(2.9)$ & $4(3.8)$ & .11 \\
\hline Extracorporeal membrane oxygenation & $0(0)$ & $1(1.0)$ & $1(0.9)$ & $>.99$ \\
\hline \multicolumn{5}{|l|}{ Pharmacologic agent, n (\%) } \\
\hline Lopinavir or ritonavir & $2(100)$ & $30(28.8)$ & $32(30.2)$ & .16 \\
\hline Chloroquine or hydroxychloroquine & $0(0)$ & $7(6.7)$ & $7(6.6)$ & $>.99$ \\
\hline Ribavirin & $0(0)$ & $0(0)$ & $0(0)$ & $>.99$ \\
\hline Remdesivir & $0(0)$ & $0(0)$ & $0(0)$ & .99 \\
\hline Oseltamivir & $0(0)$ & $2(1.9)$ & $2(1.9)$ & .99 \\
\hline Interferon & $0(0)$ & $0(0)$ & $0(0)$ & $>.99$ \\
\hline Dexamethasone & $0(0)$ & $1(1.0)$ & $1(0.9)$ & $>.99$ \\
\hline Methylprednisolone & $0(0)$ & $4(3.8)$ & $4(3.8)$ & $>.99$ \\
\hline Antibiotics & $2(100)$ & $8(7.7)$ & $10(9.4)$ & .001 \\
\hline Combination & $2(100)$ & $15(14.4)$ & $17(16.0)$ & .02 \\
\hline
\end{tabular}




\section{Discussion}

\section{Principal Findings}

Our new AI model, EDRnet, was able to predict the mortality of COVID-19 patients using 28 blood biomarkers obtained within 24 hours after hospital admission. In the independent testing data sets, EDRnet showed excellent prediction performance with high sensitivity (100\%), specificity (91\%), and accuracy $(92 \%)$. We were able to improve the prediction performance by adopting the ensemble approach combining DNN and RF models. Of note, EDRnet was developed by training with Chinese patients' data and testing with Korean patients' data.

EDRnet has several advantages. First, EDRnet can predict which patients are at a high risk of mortality in the early stage of hospital admission (ie, within 24 hours after admission). This is a substantial improvement compared to the prior AI prediction model reported by Yan et al, which predicted mortality 10 days before the occurrence of survival or death [6]. The mortality prediction at the time of admission can be substantially informative for clinicians because the critical time regarding disease progression is 10 to 14 days from the onset of symptoms, according to previous studies $[13,16,26]$. EDRnet can provide treatment priority guidance regarding who should be treated intensively. Second, EDRnet only uses blood biomarkers to predict mortality. In general, COVID-19 patients get blood laboratory tests at the time of hospital admission [9,27]. Blood biomarkers are objective indices that are used to estimate patients' conditions in a quantitative manner, which may be beneficial to assure the reliability of the AI model. We did not include subjective biomarkers, such as symptoms, nor predisposing factors, such as underlying comorbidities, because these indices are difficult for quantification and may show high variability between patients. Third, the clinical meaning and significance of blood biomarkers used in our EDRnet model have been well investigated through many prior clinical studies. Thus, the AI's predicted mortality results are explainable and easily understood by doctors. Furthermore, several major blood biomarkers are used in our EDRnet model.

The hematological changes in lymphocytes, neutrophils, monocytes, eosinophils, and platelets are common, as these changes are related to viral replication and hyperinflammation in COVID-19 infection [12,13]. In severe cases, the infiltration and sequestration of CD4+/CD8+ T cells occurred, leading to a decrease in the peripheral lymphocytes. Neutrophil counts [19-21] were significantly higher in the severe group than in the mild group. Platelet count, platelet volume, and platelet large-cell ratio are related to COVID-19 infection because immunologic destruction can lead to inappropriate platelet activation and consumption as well as impaired megakaryopoiesis [28-30].

Regarding blood chemistry, hs-CRP is a major biomarker that represents acute phase inflammation [2,10,14-17]. LDH is related to cell damage, so elevated $\mathrm{LDH}$ is an independent risk factor for the severity and mortality of COVID-19 [2,8-11].
Hypoalbuminemia [18,20,21], hypocalcemia [31-33], and elevated aspartate aminotransferase [18] are highly associated with severe COVID-19 infection requiring hospitalization in the intensive care unit. Urea and estimated glomerular filtration rate are important lab findings associated with an underlying chronic renal disease, which is a well-known predisposing factor of mortality [34]. In terms of the coagulation profile, COVID-19 generally presents a hypercoagulation state, thus resulting in an elevated prothrombin time and international normalized ratio in severe COVID-19 cases [3,18].

In this study, no significant differences were observed in the use of pharmacologic agents between the deceased and survivor groups except antibiotics and in the use of antiviral drugs, such as remdesivir. Antibiotics or combination therapy is usually used for suspected bacterial superinfection that represents severe diseases. To date, there has been no successfully effective pharmacologic agent to treat COVID-19. The pharmacologic treatment is not significantly related to survival in this study.

EDRnet does not require all 28 blood biomarkers for the prediction of mortality. EDRnet worked well as long as there were at least 19 blood biomarkers at the time of admission. Compared to prior AI prediction models for COVID-19 mortality, which used three biomarkers, there might be concern that EDRnet requires too many biomarkers. However, these blood tests are commonly performed in our daily clinical practice for hospitalized patients with COVID-19. If more data are accumulated, then we can reduce the number of blood biomarkers for mortality prediction.

\section{Limitations and Future Work}

Our study has several limitations. First, the number of patients available for testing might be small. According to Johns Hopkins Coronavirus Resource Center, the mortality rate in South Korea is $1.7 \%$. In the testing data set of 106 Korean patients, the mortality rate was $1.9 \%$, which is almost equivalent to the actual mortality rate. It might be necessary to update EDRnet by training with a large population data set from all over the world. To update EDRnet, we made a web application [25] so that anyone can access the model. We believe that opening the AI model to the public is helpful to improve its performance and generalizability. Second, our data did not include other races, such as Caucasian or Middle East Asian. Our future research plan is to establish a real-time AI training system that can continue to train our model using prospectively collected data from all over the world. In addition, we will upgrade the web application so that the database framework allows a user to input his or her blood sample results along with the outcome. Based on the extended data, we will improve EDRnet for better generalization.

\section{Conclusions}

In conclusion, our new AI model, EDRnet, was developed to predict the mortality of COVID-19 patients at the time of hospital admission using blood biomarkers only. It is now open to the public with the hope that it can help health care providers fight COVID-19 and improve patients' outcomes. 


\section{Acknowledgments}

This work was supported by Korea Medical Device Development Fund grants, funded by the Korean government (the Ministry of Science and ICT; the Ministry of Trade, Industry and Energy; the Ministry of Health and Welfare, Republic of Korea; and the Ministry of Food and Drug Safety) (grant Nos. 202012B04 and NRF-2020R1A2C1014829), and by the Korea Health Industry Development Institute (grant No. HI18C1216). Author RJ is currently an MIT Lincoln Laboratory employee. No Laboratory funding or resources were used to produce the result/findings reported in this publication.

\section{Authors' Contributions}

$\mathrm{HK}$ and $\mathrm{HC}$ carried out the machine learning and deep learning simulation for hyperparameter search and modeling. CP verified blood biomarkers to be applied in COVID-19 patients and wrote an initial draft of the paper. DWK, SEK, CRC, and REK collected and validated the data and performed statistical analyses. HL developed and maintained the web application. KWK, JHS, and TYC validated and confirmed the simulations and helped to draft the manuscript. RJ validated the methodology of machine learning and deep learning techniques. JL and WSK conceived of the study and participated in its design and coordination and wrote the initial manuscript. All authors read and approved the final manuscript.

\section{Conflicts of Interest}

None declared.

\section{Multimedia Appendix 1}

Training data set.

[XLSX File (Microsoft Excel File), 96 KB-Multimedia Appendix 1]

\section{Multimedia Appendix 2}

Testing data set.

[XLSX File (Microsoft Excel File), 35 KB-Multimedia Appendix 2]

\section{Multimedia Appendix 3}

Supplementary figures and tables.

[DOCX File, 876 KB-Multimedia Appendix 3]

\section{References}

1. COVID-19 Surveillance Group. Characteristics of COVID-19 Patients Dying in Italy: Report Based on Available Data on March 20th, 2020. Rome, Italy: Istituto Superiore di Sanità; 2020. URL: https://www.epicentro.iss.it/coronavirus/bollettino/ Report-COVID-2019 20 marzo eng.pdf [accessed 2020-12-17]

2. $\quad$ Li C, Ye J, Chen Q, Hu W, Wang L, Fan Y, et al. Elevated lactate dehydrogenase (LDH) level as an independent risk factor for the severity and mortality of COVID-19. Aging (Albany NY) 2020 Aug 14;12(15):15670-15681 [FREE Full text] [doi: 10.18632/aging.103770] [Medline: 32805722]

3. Luo L, Xu M, Du M, Kou H, Liao D, Cheng Z, et al. Early coagulation tests predict risk stratification and prognosis of COVID-19. Aging (Albany NY) 2020 Aug 29;12(16):15918-15937 [FREE Full text] [doi: 10.18632/aging.103581] [Medline: 32860672]

4. Lai C, Liu YH, Wang C, Wang Y, Hsueh S, Yen M, et al. Asymptomatic carrier state, acute respiratory disease, and pneumonia due to severe acute respiratory syndrome coronavirus 2 (SARS-CoV-2): Facts and myths. J Microbiol Immunol Infect 2020 Jun;53(3):404-412 [FREE Full text] [doi: 10.1016/j.jmii.2020.02.012] [Medline: 32173241]

5. Sohrabi C, Alsafi Z, O'Neill N, Khan M, Kerwan A, Al-Jabir A, et al. World Health Organization declares global emergency: A review of the 2019 novel coronavirus (COVID-19). Int J Surg 2020 Apr;76:71-76 [FREE Full text] [doi:

10.1016/j.ijsu.2020.02.034] [Medline: $\underline{32112977]}$

6. Yan L, Zhang H, Goncalves J, Xiao Y, Wang M, Guo Y, et al. An interpretable mortality prediction model for COVID-19 patients. Nat Mach Intell 2020 May 14;2(5):283-288. [doi: 10.1038/s42256-020-0180-7]

7. Chen T, Guestrin C. XGBoost: A scalable tree boosting system. In: Proceedings of the 22nd ACM SIGKDD International Conference on Knowledge Discovery and Data Mining. New York, NY: Association for Computing Machinery; 2016 Presented at: 22nd ACM SIGKDD International Conference on Knowledge Discovery and Data Mining; August 13-17, 2016; San Francisco, CA p. 785-794 URL: https://dl.acm.org/doi/pdf/10.1145/2939672.2939785 [doi: $10.1145 / 2939672.2939785]$

8. Wu MY, Yao L, Wang Y, Zhu XY, Wang XF, Tang PJ, et al. Clinical evaluation of potential usefulness of serum lactate dehydrogenase (LDH) in 2019 novel coronavirus (COVID-19) pneumonia. Respir Res 2020 Jul 06;21(1):171 [FREE Full text] [doi: 10.1186/s12931-020-01427-8] [Medline: 32631317$]$ 
9. Henry BM, de Oliveira MHS, Benoit S, Plebani M, Lippi G. Hematologic, biochemical and immune biomarker abnormalities associated with severe illness and mortality in coronavirus disease 2019 (COVID-19): A meta-analysis. Clin Chem Lab Med 2020 Jun 25;58(7):1021-1028. [doi: 10.1515/cclm-2020-0369] [Medline: 32286245]

10. Henry BM, Aggarwal G, Wong J, Benoit S, Vikse J, Plebani M, et al. Lactate dehydrogenase levels predict coronavirus disease 2019 (COVID-19) severity and mortality: A pooled analysis. Am J Emerg Med 2020 Sep;38(9):1722-1726 [FREE Full text] [doi: 10.1016/j.ajem.2020.05.073] [Medline: $\underline{\text { 32738466] }}$

11. Panteghini M. Lactate dehydrogenase: An old enzyme reborn as a COVID-19 marker (and not only). Clin Chem Lab Med 2020 Aug 24;58(12):1979-1981. [doi: 10.1515/cclm-2020-1062] [Medline: 32829312]

12. Huang I, Pranata R. Lymphopenia in severe coronavirus disease-2019 (COVID-19): Systematic review and meta-analysis. J Intensive Care 2020;8:36 [FREE Full text] [doi: 10.1186/s40560-020-00453-4] [Medline: 32483488]

13. Ruan Q, Yang K, Wang W, Jiang L, Song J. Clinical predictors of mortality due to COVID-19 based on an analysis of data of 150 patients from Wuhan, China. Intensive Care Med 2020 May;46(5):846-848 [FREE Full text] [doi: 10.1007/s00134-020-05991-x] [Medline: 32125452]

14. Wang L. C-reactive protein levels in the early stage of COVID-19. Med Mal Infect 2020 Jun;50(4):332-334 [FREE Full text] [doi: 10.1016/j.medmal.2020.03.007] [Medline: 32243911]

15. Wang D, Hu B, Hu C, Zhu F, Liu X, Zhang J, et al. Clinical characteristics of 138 hospitalized patients with 2019 novel coronavirus-infected pneumonia in Wuhan, China. JAMA 2020 Mar 17;323(11):1061-1069 [FREE Full text] [doi: 10.1001/jama.2020.1585] [Medline: $\underline{32031570]}$

16. Guan W, Ni Z, Hu Y, Liang W, Ou C, He J, China Medical Treatment Expert Group for Covid-19. Clinical characteristics of coronavirus disease 2019 in China. N Engl J Med 2020 Apr 30;382(18):1708-1720 [FREE Full text] [doi: 10.1056/NEJMoa2002032] [Medline: 32109013]

17. Tan C, Huang Y, Shi F, Tan K, Ma Q, Chen Y, et al. C-reactive protein correlates with computed tomographic findings and predicts severe COVID-19 early. J Med Virol 2020 Jul;92(7):856-862 [FREE Full text] [doi: 10.1002/jmv.25871] [Medline: $\underline{\text { 32281668] }}$

18. Long H, Nie L, Xiang X, Li H, Zhang X, Fu X, et al. D-dimer and prothrombin time are the significant indicators of severe COVID-19 and poor prognosis. Biomed Res Int 2020;2020:6159720 [FREE Full text] [doi: 10.1155/2020/6159720] [Medline: 32596339]

19. Kong M, Zhang H, Cao X, Mao X, Lu Z. Higher level of neutrophil-to-lymphocyte is associated with severe COVID-19. Epidemiol Infect 2020 Jul 09;148:e139 [FREE Full text] [doi: 10.1017/S0950268820001557] [Medline: 32641174]

20. Aziz M, Fatima R, Lee-Smith W, Assaly R. The association of low serum albumin level with severe COVID-19: A systematic review and meta-analysis. Crit Care 2020 May 26;24(1):255 [FREE Full text] [doi: 10.1186/s13054-020-02995-3] [Medline: 32456658]

21. Aziz M, Fatima R, Assaly R. Elevated interleukin-6 and severe COVID-19: A meta-analysis. J Med Virol 2020 Nov;92(11):2283-2285 [FREE Full text] [doi: 10.1002/jmv.25948] [Medline: 32343429]

22. The Lancet Haematology. COVID-19 coagulopathy: An evolving story. Lancet Haematol 2020 Jun;7(6):e425 [FREE Full text] [doi: 10.1016/S2352-3026(20)30151-4] [Medline: $\underline{\text { 32470428] }}$

23. Arachchillage DRJ, Laffan M. Abnormal coagulation parameters are associated with poor prognosis in patients with novel coronavirus pneumonia. J Thromb Haemost 2020 May;18(5):1233-1234. [doi: 10.1111/jth.14820] [Medline: 32291954]

24. Bowles L, Platton S, Yartey N, Dave M, Lee K, Hart DP, et al. Lupus anticoagulant and abnormal coagulation tests in patients with COVID-19. N Engl J Med 2020 Jul 16;383(3):288-290 [FREE Full text] [doi: 10.1056/NEJMc2013656] [Medline: $\underline{\text { 32369280] }}$

25. BeatCOVID19. URL: http://beatcovid19.ml/ [accessed 2020-12-15]

26. Qian G, Yang N, Ding F, Ma AHY, Wang Z, Shen Y, et al. Epidemiologic and clinical characteristics of 91 hospitalized patients with COVID-19 in Zhejiang, China: A retrospective, multi-centre case series. QJM 2020 Jul 01;113(7):474-481 [FREE Full text] [doi: 10.1093/qjmed/hcaa089] [Medline: $\underline{\text { 32181807] }}$

27. Luo Y, Yuan X, Xue Y, Mao L, Lin Q, Tang G, et al. Using a diagnostic model based on routine laboratory tests to distinguish patients infected with SARS-CoV-2 from those infected with influenza virus. Int J Infect Dis 2020 Jun;95:436-440 [FREE Full text] [doi: 10.1016/j.ijid.2020.04.078] [Medline: 32371192]

28. Zhao X, Wang K, Zuo P, Liu Y, Zhang M, Xie S, et al. Early decrease in blood platelet count is associated with poor prognosis in COVID-19 patients-Indications for predictive, preventive, and personalized medical approach. EPMA J 2020 May 14:1-7 [FREE Full text] [doi: 10.1007/s13167-020-00208-z] [Medline: $\underline{\text { 32419876] }}$

29. Zhu S, Dong L, Cai W. Predictive value of neutrophil to lymphocyte and platelet to lymphocyte ratio in COVID-19. Crit Care 2020 Aug 28;24(1):532 [FREE Full text] [doi: 10.1186/s13054-020-03258-x] [Medline: 32859254]

30. Sadr S, SeyedAlinaghi S, Ghiasvand F, Hassan Nezhad M, Javadian N, Hossienzade R, et al. Isolated severe thrombocytopenia in a patient with COVID-19: A case report. IDCases 2020;21:e00820 [FREE Full text] [doi: 10.1016/j.idcr.2020.e00820] [Medline: 32483524]

31. Cappellini F, Brivio R, Casati M, Cavallero A, Contro E, Brambilla P. Low levels of total and ionized calcium in blood of COVID-19 patients. Clin Chem Lab Med 2020 Aug 27;58(9):e171-e173. [doi: 10.1515/cclm-2020-0611] [Medline: 32459190] 
32. Di Filippo L, Formenti AM, Rovere-Querini P, Carlucci M, Conte C, Ciceri F, et al. Hypocalcemia is highly prevalent and predicts hospitalization in patients with COVID-19. Endocrine 2020 Jun;68(3):475-478 [FREE Full text] [doi: 10.1007/s12020-020-02383-5] [Medline: 32533508]

33. Sun J, Zhang W, Zou L, Liu Y, Li J, Kan X, et al. Serum calcium as a biomarker of clinical severity and prognosis in patients with coronavirus disease 2019. Aging (Albany NY) 2020 Jun 25;12(12):11287-11295 [FREE Full text] [doi: 10.18632/aging.103526] [Medline: 32589164]

34. Gao M, Wang Q, Wei J, Zhu Z, Li H. Severe coronavirus disease 2019 pneumonia patients showed signs of aggravated renal impairment. J Clin Lab Anal 2020 Oct;34(10):e23535 [FREE Full text] [doi: 10.1002/jcla.23535] [Medline: 32840917]

\author{
Abbreviations \\ AB: AdaBoost \\ ADR: available data rate \\ AI: artificial intelligence \\ ANOVA: analysis of variance \\ CNUH: Chonnam National University Hospital \\ DNN: deep neural network \\ EDA: ensemble with deep neural network and AdaBoost \\ EDRnet: ensemble learning model based on deep neural network and random forest models \\ EDX: ensemble with deep neural network and XGBoost \\ FC: fully connected \\ FN: false negative \\ FP: false positive \\ GPU: graphics processing unit \\ hs-CRP: high-sensitivity C-reactive protein \\ LDH: lactate dehydrogenase \\ RF: random forest \\ SMC: Samsung Medical Center \\ TN: true negative \\ TP: true positive \\ WKUH: Wonkwang University Hospital
}

\author{
Edited by G Eysenbach, $R$ Kukafka; submitted 03.11.20; peer-reviewed by J Lee, C Jeong; comments to author 24.11.20; revised \\ version received 24.11.20; accepted 08.12.20; published 23.12.20 \\ Please cite as: \\ Ko H, Chung H, Kang WS, Park C, Kim DW, Kim SE, Chung CR, Ko RE, Lee H, Seo JH, Choi TY, Jaimes R, Kim KW, Lee J \\ An Artificial Intelligence Model to Predict the Mortality of COVID-19 Patients at Hospital Admission Time Using Routine Blood \\ Samples: Development and Validation of an Ensemble Model \\ J Med Internet Res 2020;22(12):e25442 \\ URL: http://www.jmir.org/2020/12/e25442/ \\ doi: $10.2196 / 25442$ \\ PMID: 33301414
}

CHoon Ko, Heewon Chung, Wu Seong Kang, Chul Park, Do Wan Kim, Seong Eun Kim, Chi Ryang Chung, Ryoung Eun Ko, Hooseok Lee, Jae Ho Seo, Tae-Young Choi, Rafael Jaimes, Kyung Won Kim, Jinseok Lee. Originally published in the Journal of Medical Internet Research (http://www.jmir.org), 23.12.2020. This is an open-access article distributed under the terms of the Creative Commons Attribution License (https://creativecommons.org/licenses/by/4.0/), which permits unrestricted use, distribution, and reproduction in any medium, provided the original work, first published in the Journal of Medical Internet Research, is properly cited. The complete bibliographic information, a link to the original publication on http://www.jmir.org/, as well as this copyright and license information must be included. 